\title{
Percepções sobre a Rede de Cuidados à Pessoa com Deficiência em uma Região de Saúde
}

\section{Perceptions on the Care Network for Disabled Persons in a Health Region}

Camila Dubow' ${ }^{\mathbf{1}}$ Edna Linhares Garcia², Suzane Beatriz Frantz Krug $\mathbf{3}$
1 Universidade de Santa Cruz do Sul (Unisc) - Santa Cruz do Sul (RS), Brasil. camiladubow@yahoo.com.br

2 Universidade de Santa Cruz do Sul (Unisc) - Santa Cruz do Sul (RS), Brasil. edna@unisc.br

3 Universidade de Santa Cruz do Sul (Unisc) - Santa Cruz do Sul (RS), Brasil. skrug@unisc.br
RESUMO O objetivo do estudo foi o de analisar o processo de implantação da Rede de Cuidados à Pessoa com Deficiência em uma Região de Saúde. Por meio de pesquisa exploratória, realizou-se um estudo de caso empregando questionários e entrevistas com 49 sujeitos, de forma a mapear, descrever e analisar o contexto, relações e percepções de distintos atores envolvidos no processo de implantação da referida rede. Os dados revelaram a existência de movimentos que denunciam uma 'rede viva de cuidados' em permanente construção, em processo gradual de incorporação de suas diretrizes, embora ainda se enfatizem os serviços especializados de reabilitação como centralizadores do cuidado.

PalaVRas-ChaVe Sistema Único de Saúde. Pessoas com deficiência. Assistência à saúde. Regionalização. Integralidade em saúde.

ABSTRACT The objective of this study was to analyze the implementation process of the network for disabled people in a regional health system in the State of Rio Grande do Sul, Brazil. An exploratory study was used to conduct a case study, which applied questionnaires and interviews with 49 individuals so to map, describe and analyze the context, relations and perceptions) of different actors involved in the network implementation process. The results revealed the existence of movements reporting a 'living healthcare network' in permanent construction, following a gradual process of guidelines incorporation, although specialized rehabilitation services are still emphasized as care centralizers.

KEYWORDS Unified Health System. Disabled persons. Delivery of health care. Regional health planning. Integrality in health. 


\section{Introdução}

O direito universal à saúde, consolidado a partir do Sistema Único de Saúde (SUS), possibilitou a incorporação das necessidades das Pessoas com Deficiência $(\mathrm{PcD})$ às políticas públicas de saúde. Porém, historicamente, a maioria das iniciativas no campo da atenção à saúde voltada a esse público tem sido isolada e em desacordo com os princípios de integralidade, equidade e acesso qualificado e universal à saúde, não promovendo uma articulação consistente entre os pontos e níveis de atenção à saúde, resultando em atenção fragmentada e pouco eficaz'

Mais de um bilhão de pessoas em todo o mundo apresentam algum tipo de deficiência, sendo que cerca de 200 milhões experimentam dificuldades funcionais consideráveis ${ }^{2}$. De acordo com o Censo realizado pelo Instituto Brasileiro de Geografia e Estatística (IBGE) em 2010, 23,9\% da população brasileira declarou possuir algum tipo de deficiência, sendo $23,8 \%$ no Rio Grande do Sul. Na $28^{\text {a }}$ Região de Saúde desse Estado, 30,81\% da população declarou algum tipo de deficiência, sendo mais frequente a deficiência visual $(16,26 \%)$, seguida pelas deficiências motora ou física (7,73\%), auditiva $(5,41 \%)$ e mental ou intelectual $(1,41 \%)^{3}$.

Assim, tem-se o desafio de concretizar uma política pública capaz de responder de modo ampliado às necessidades de saúde dessa população, que articule os distintos níveis de atenção, visando à integralidade por meio de cuidado em rede que atravesse os diversos serviços de saúde, implicando conexões e comunicações fundamentais para o bom desempenho do SUS ${ }^{4,5}$.

Para isso, o cuidado às pessoas com deficiência deve ser pensado em Redes de Atenção à Saúde (RAS) ${ }^{\mathbf{6}}$ em função das necessidades da população, das demandas regionais e dos arranjos territoriais, permitindo que a atenção em saúde esteja focada na realidade do território e que dê sustentação a projetos terapêuticos singulares, ampliando as possibilidades de equidade e de cuidado integral a estas pessoas' ${ }^{1}$.
Neste contexto, foi instituída, por meio da Portaria $n^{0} 793$ de 24 de abril de 2012, a Rede de Cuidados à Pessoa com Deficiência (RCPCD) no âmbito do SUS com o objetivo de ampliar o acesso, qualificar o atendimento em saúde, promover a vinculação das $\mathrm{PcD}$ e suas famílias aos pontos de atenção à saúde e garantir a articulação e a integração desses pontos nos territórios ${ }^{7}$.

A implantação dessa política como meio de ampliar a capacidade de acolhimento, cuidado à saúde e qualidade de vida das PcD exige pesquisas, debates e reflexões contínuas sobre os desenhos da rede nas diferentes realidades regionais frente à diversificação e às dimensões do Brasil8. Desse modo, estudar seu processo de implantação em uma Região de Saúde específica justifica-se pelas singularidades das RAS nos diferentes territórios. Assim, para que a atenção à saúde das $\mathrm{PcD}$ seja qualificada, torna-se fundamental conhecer a realidade, promover a discussão e reflexão sobre o tema, a fim de fornecer elementos para o planejamento de ações e definição de prioridades para implantação de políticas públicas. Desta maneira, possibilita-se o preparo do sistema de saúde para o enfrentamento qualificado das deficiências, além de contribuir para a valorização da coordenação e continuidade do cuidado? .

Desse modo, como referencial teórico norteador deste estudo, adotaram-se as RAS, definidas pela Portaria ${ }^{\circ} 4.279 / 10$ como:

arranjos organizativos de ações e serviços de saúde, de diferentes densidades tecnológicas, que integradas por meio de sistemas de apoio técnico, logístico e de gestão, buscam garantir a integralidade do cuidado ${ }^{\mathbf{6 ( 8 8 )}}$.

Resumindo, o objetivo deste estudo foi o de analisar o processo de implantação da RCPCD na $28^{\text {a }}$ Região de Saúde do Rio Grande do Sul, dada a necessidade de o Brasil conhecer as particularidades de suas regiões para fortalecer e qualificar a atenção à saúde das pessoas com deficiência. 


\section{Métodos}

Realizou-se um estudo de caso por meio de pesquisa exploratória que empregou estratégias de investigação quantitativa e qualitativa para mapear, descrever e analisar o contexto, relações e percepções a respeito do tema ${ }^{10}$.

A pesquisa foi realizada na $28^{\mathrm{a}}$ Região de Saúde do Rio Grande do Sul, localizada na região central desse Estado, onde vive uma população de 327.158 habitantes distribuída em treze municípios. A rede de atenção à saúde da região oferece desde a atenção básica à saúde até as média e alta complexidades e está organizada em 51 Estratégias Saúde da Família (ESF), 44 Unidades Básicas de Saúde (UBS), nove equipes de Núcleo de Apoio à Saúde da Família (Nasf), um serviço de reabilitação física, dois serviços de reabilitação intelectual e três hospitais gerais com 80 leitos ou mais.

A amostra foi composta por 60 sujeitos pertencentes a sete segmentos envolvidos na atenção à saúde das pessoas com deficiência e que representam os diferentes níveis de participação nos distintos contextos municipais e regional, permitindo melhor apreensão da rede sob diferentes perspectivas, conforme a realidade da qual os sujeitos falam ou atuam. Houve exclusão de onze sujeitos, devido a não devolução do instrumento de coleta. Considerou-se, em amostragem intencional, um conjunto de informantes que possibilitou a apreensão de semelhanças e diferenças nas respostas, bem como um número de sujeitos suficiente para permitir a reincidência das informações ${ }^{\mathbf{1 0}}$, conforme ilustrado na figura 1:

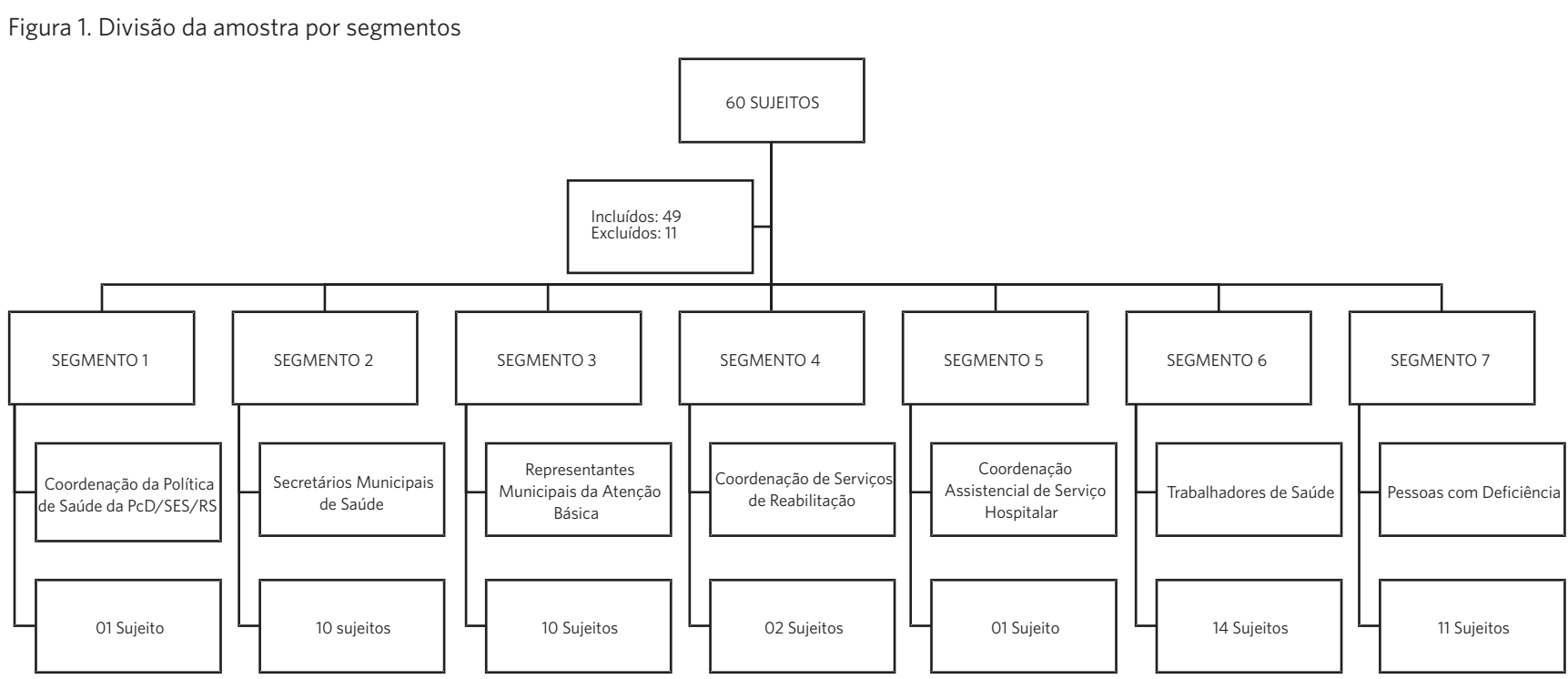

Os sujeitos dos segmentos um ao cinco eram os titulares dos cargos ou seus representantes legais quando da coleta de dados. Para o segmento seis, foram selecionados representantes de cada componente da RCPCD, sendo onze trabalhadores da atenção básica, dois de serviços de reabilitação e um de instituição hospitalar. Os sujeitos do segmento sete foram indicados pelos Conselhos Municipais dos Direitos da 
Pessoa com Deficiência (Compede) ou coordenadores de serviços de reabilitação.

Para a coleta de dados, realizada de maio a outubro de 2016, foram utilizados dois instrumentos em momentos distintos, diferenciados para cada segmento de sujeitos: questionários e entrevistas semiestruturadas. Inicialmente, foi feito teste piloto com representantes de cada segmento para avaliação dos instrumentos quanto à sua apresentação e compreensão, não havendo necessidade de adequações. Após, foram aplicados os questionários com o objetivo de elaborar um panorama geral referente à implantação da RCPCD com Deficiência na 28a Região de Saúde. Em seguida, foram realizadas entrevistas semiestruturadas com doze sujeitos, gravadas em mídia digital mediante consentimento prévio e transcritas em sua íntegra.

Para a análise, os dados dos questionários foram classificados em frequências absolutas e relativas, tendo a análise de dados das entrevistas sido feita por meio da técnica de análise de conteúdo, com apresentação de trechos das entrevistas realizadas. Conforme preconiza a análise de conteúdo, este estudo cumpriu as etapas de pré-análise com leitura compreensiva dos textos transcritos; exploração do material visando a alcançar o núcleo de compreensão do texto por meio da elaboração de categorias de análise e agrupar trechos de depoimentos significativos; e tratamento dos resultados obtidos e interpretação dos dados ${ }^{\mathbf{1 0}}$.

Esse processo culminou em duas categorias temáticas. A primeira, denominada Movimentos de uma Rede Viva de Cuidados nos Territórios, permitiu a análise e reflexão sobre as potências, limitações e perspectivas da RCPCD na região estudada buscando tensionar as diretrizes propostas pela política que institui a $\mathrm{RCPCD}^{7}$. Tal análise deu origem às subcategorias (i) Estrutura Operacional e Modelo de Atenção, que apresenta a conformação da RCPCD na região estudada, com enfoque para a sua estrutura operacional e o modelo de atenção vigente ou predominante; e (ii) Fluxos Assistenciais e Articulação da Rede, que apresenta os fluxos assistenciais existentes na RCPCD da região estudada, com enfoque para os mecanismos de sua articulação.

A segunda categoria, intitulada (Im) possibilidades da Rede, refletiu sobre as potências, limitações e perspectivas da RCPCD na região estudada. Ressalta-se que, ao mesmo tempo em que algumas questões foram indicadas como potências por alguns sujeitos, também surgiram como limitações para outros, quando olhadas sob outra perspectiva.

Reforça-se, ainda, a inter-relação entre as duas categorias de análise, apresentadas de maneira isolada neste texto para melhor compreensão dos aspectos fundamentais abordados em cada uma delas. Contudo, destaca-se que a conformação da rede, apresentada e discutida na primeira categoria, está diretamente relacionada aos limites e potencialidades apresentados na segunda categoria, não podendo, portanto, ser entendidas de maneira isolada, assim como são as redes vivas de permanente ir e vir, de construção e desconstrução de suas interfaces.

Em cumprimento aos requisitos éticos da Resolução $n^{\circ}$ 466/2012 do Conselho Nacional de Saúde, o projeto foi aprovado pelo Comitê de Ética em Pesquisa da Universidade de Santa Cruz do Sul, sob o protocolo 1.300.666/15, e todos os participantes assinaram o Termo de Consentimento Livre e Esclarecido. Para garantir o anonimato dos participantes, as narrativas foram identificadas com o uso de letras, acompanhadas de numeração por segmentos, conforme figura 1.

\section{Resultados e discussão}

Predominaram no estudo sujeitos do sexo feminino $(71,5 \%)$ com idade entre 29 e 50 anos (59,19\%), ensino superior (24,5\%), pós-graduação (47\%), conforme perfil apresentado na tabela 1. 
Tabela 1. Caracterização dos sujeitos do estudo de acordo com os 7 segmentos

\begin{tabular}{|c|c|c|c|c|c|c|c|c|}
\hline \multirow{2}{*}{ SEGMENTO } & 1 & 2 & 3 & 4 & 5 & 6 & 7 & TOTAL \\
\hline & n (\%) & n (\%) & n (\%) & n (\%) & n (\%) & n (\%) & n (\%) & n (\%) \\
\hline Feminino & $1(100)$ & $6(60)$ & $10(100)$ & $2(100)$ & $1(100)$ & $11(78,5)$ & $4(36,36)$ & $35(71,5)$ \\
\hline Masculino & & $4(40)$ & & & & $3(21,5)$ & $7(63,64)$ & $14(28,5)$ \\
\hline \multicolumn{9}{|l|}{ Idade (anos) } \\
\hline $0-17$ & & & & & & & $4(36,36)$ & $4(8,1)$ \\
\hline $18-28$ & & & $1(10)$ & & & $3(21,5)$ & $1(9,09)$ & $5 \mid(10)$ \\
\hline $29-39$ & & $2(20)$ & $6(60)$ & & $1(100)$ & $5(35,7)$ & $2(18,18)$ & $16(33)$ \\
\hline 40 a 50 & & $4(40)$ & $2(20)$ & & & $5(35,7)$ & $2(18,18)$ & $13(26,5)$ \\
\hline 51 a 60 & $1(100)$ & $1(10)$ & $1(10)$ & $2(100)$ & & $1(7,14)$ & $1(9,09)$ & $7(14,3)$ \\
\hline Mais de 60 & & $3(30)$ & & & & & $1(9,09)$ & $4(8,10)$ \\
\hline \multicolumn{9}{|l|}{ Escolaridade } \\
\hline Não Escolarizado & & & & & & & $3(27,27)$ & $3(6,1)$ \\
\hline Ensino Fundamental & & & & & & & $5(45,45)$ & $5(10,2)$ \\
\hline Ensino Médio & & $3(30)$ & & & & & $3(27,27)$ & $6(12,2)$ \\
\hline Ensino Superior & & $4(40)$ & $2(20)$ & & & $6(42,85)$ & & $12(24,5)$ \\
\hline Pós-Graduação & 1 & $3(30)$ & $8(80)$ & $2(100)$ & $1(100)$ & $8(57,15)$ & & $23(47)$ \\
\hline TOTAL & 01 & 10 & 10 & 02 & 01 & 14 & 11 & 49 \\
\hline
\end{tabular}

\section{Movimentos de uma Rede Viva de Cuidados nos Territórios}

Entende-se por rede viva o modo com que se produzem as conexões de indivíduos e coletivos, em diferentes contextos de grupalidade e modos de viver socialmente ${ }^{\mathbf{1 1}}$. Sob essa perspectiva, vislumbrou-se conhecer ou reconhecer as infinitas e mutantes formas de conexão na RCPCD estudada e, assim, abrir-se para a criação de múltiplas formas de produção de cuidado e acolhimento, trazendo para a cena toda o radicalismo do campo da vida e da ética, ao tipo de aposta que a rede de cuidados afirma e tensiona ${ }^{12}$.

\section{ESTRUTURA OPERACIONAL E MODELO DE ATENĈ̣̃O}

Em relação à estrutura operacional, essa rede constitui-se por diferentes pontos de atenção à saúde, com enfoque nos serviços especializados de reabilitação como centralizadores do cuidado. Na região estudada, a assistência em saúde às pessoas com deficiências física e intelectual possui serviços especializados de referência com sede na própria região de saúde, enquanto a assistência às pessoas com deficiências auditiva e visual apresenta serviços de referência em outras regiões de saúde, conforme demonstrado na figura 2: 
Figura 2. Estrutura Operacional da RCPCD na 28a Região de Saúde, RS

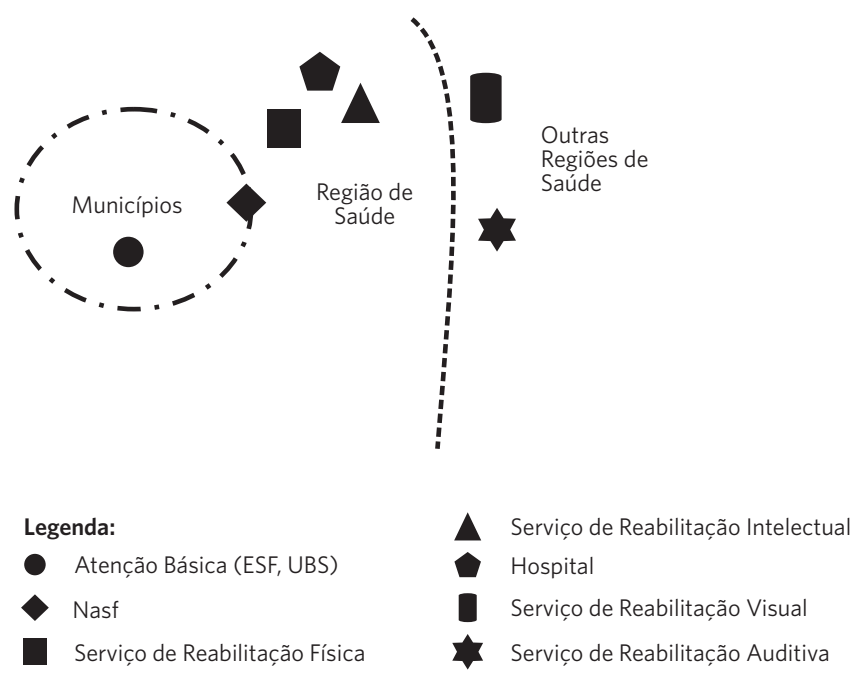

A proposta da RCPCD no âmbito do SUS prevê que os cuidados a essas pessoas devam ser organizados a partir de três componentes: Atenção Básica, Atenção Especializada em Reabilitação, Atenção Hospitalar, Urgência e Emergência, que, articulados entre si, garantem a integralidade do cuidado e o acesso regulado aos pontos de atenção7. Assim, conforme figura 2, é possível perceber que esses três componentes estão presentes na RCPCD da região estudada.

No que tange às ações direcionadas às $\mathrm{PcD}$ na região, dentre os gestores municipais, coordenadores de serviços da Atenção Básica, Especializada e Hospitalar e trabalhadores de saúde, $76 \%$ responderam que realizam ou são realizadas ações em saúde voltadas às $\mathrm{PcD}$ em seus respectivos serviços ou municípios, enquanto $19 \%$ responderam não e $5 \%$ não souberam responder. Dentre os que afirmaram a existência de ações, as mais citadas foram, respectivamente, reabilitação (69\%) e tratamento (56\%), seguidas por ações de promoção à saúde (47\%), prevenção de doenças (44\%), educação em saúde (44\%) e outras ações (19\%).

Emergiu, a partir da análise dos dados, um movimento para a reorganização da atenção que contemple a integralidade e considere as vulnerabilidades desse grupo ou população e suas necessidades, conforme diretrizes preconizadas para as RAS6. Ao mesmo tempo, alguns sujeitos informaram a inexistência de ações voltadas especificamente para a saúde das PcD, ilustrada pelo trecho de entrevista a seguir: "Ações específicas, não. Não existe um programa de atendimento específico para eles [PcD]" (B1).

Identificou-se, também, que, embora muitos sujeitos, ao responderem de imediato sobre a inexistência de tais atividades, perceberam, no decorrer da coleta de dados que, mesmo que não instituídas ou formalmente caracterizadas, são realizadas diversas ações no âmbito da atenção à saúde desses sujeitos, produzindo, assim, novos sentidos à sua prática profissional ou de gestão, conforme ilustrado pelo depoimento:

A gente não tem um protocolo pronto no papel, mas as ações são feitas, quando tu te dá conta ela tá sendo realizada. Só que a gente não se dá conta que ela já existe. Temos, só que ela tá ali, no dia a dia, essa prioridade existe. (S1). 
A esse respeito, é comum que os trabalhadores de saúde, gestores e coordenadores de serviços estejam promovendo o cuidado das $\mathrm{PcD}$, mas, paradoxalmente, não percebam isso, evidenciando, dessa maneira, a construção de uma rede por meio de movimentos que permeiem o cotidiano dos encontros. Desse modo, percebe-se a criação de arranjos singulares na conformação da rede que pretendem abranger as necessidades dos usuários, levando em conta o fato que, como uma rede viva de cuidados, a RCPCD na região estudada está se estabelecendo a partir de suas necessidades locais ou territoriais e em função das características locais ou regionais presentes.

Sobre o modelo de atenção adotado, ficou evidente o predomínio de um modelo hegemônico, centrado na doença e na oferta de serviços e procedimentos. Assim como em estudo que analisou o papel da atenção primária no cuidado às $\mathrm{PcD}^{\mathbf{1 3}}$, percebeu-se, na realidade estudada, o imaginário de que essas pessoas devem ser acompanhadas, predominantemente, por serviços especializados de reabilitação, o que desconsidera o sujeito e sua singularidade independentemente da deficiência.

Esses centros especializados são pontos de atenção para ações específicas, além de espaços de articulação com os outros pontos de atenção do SUS, caracterizando-se, assim, como o 'nós da rede'. São estratégicos para a qualificação, regulação e criação de padrões mínimos para os cuidados às $\mathrm{PcD}$, inclusive no que se refere ao acolhimento das diferenças e da humanização da atençãol. Entretanto, a procura crescente por tais serviços gera extensas filas de espera e, por consequência, demora no atendimento ou até mesmo dificuldades ou barreiras de acesso às ações e serviços de reabilitação.

A proposta descrita pela Portaria $n^{0}$ 793/2012, que instituiu a RCPCD no âmbito do SUS7, torna-se uma possibilidade de construção de novas práticas de atenção em diferentes cenários. Nessa direção, percebe-se o movimento, principalmente dos trabalhadores da saúde, em estabelecer novas conexões para trabalhar multiplicidades e trazer para o campo do cuidado as singularidades dos sujeitos, como uma 'rede viva de cuidados' em constante produçãon'.

\section{FLUXOS ASSISTENCIAIS E ARTICULAC̣ÃO DA REDE}

Dentre os sujeitos questionados, $59 \%$ afirmaram conhecer algum instrumento que oriente o fluxo dos usuários na rede, sendo os mais citados os protocolos de encaminhamentos (38\%) e os documentos de referência e contra referência (29\%).

A maioria dos fluxos assistenciais se encontra fundamentada na prática cotidiana do encaminhamento, que ocorre quando um ponto da rede não consegue contemplar as necessidades dos usuários:

Eles são atendidos na UBS [Unidade Básica de Saúde] e se não for possível o atendimento aqui, os profissionais fazem o devido encaminhamento de acordo com as referências. (B1).

Os mecanismos de referência e contra referência são modos de organização dos serviços por meio da configuração de redes sustentadas por critérios, fluxos e mecanismos de pactuação de funcionamento. Nessa compreensão, deve-se reafirmar a perspectiva de seu desenho lógico, permitindo encaminhamentos resolutivos entre os diferentes equipamentos de saúde e, ao mesmo tempo, fomentando vínculos em diferentes dimensões: intraequipes de saúde, interequipes ou serviços, entre trabalhadores e gestores, e entre usuários e serviços ou equipes ${ }^{14}$.

Assim, entende-se que a resolução das necessidades de saúde das PcD são importantes para os sujeitos da pesquisa, e estes se movimentam em busca de alternativas para que isso se efetive por meio de estratégias de articulação dos diversos pontos da rede de atenção. Entretanto, a trajetória dessas 
pessoas nos serviços de saúde ainda está focada, mais do que em suas necessidades de saúde, na oferta de serviços e procedimentos disponíveis, culminando em uma atenção muitas vezes fragmentada e que não garante a continuidade e integralidade do cuidado. A maneira pela qual os formulários de encaminhamentos e os documentos de referência e contra referência são utilizados refletem um modelo hierarquizado que não favorece a relação interprofissional e o trabalho em rede.

No que diz respeito às dificuldades nos fluxos assistenciais, a falta de integração entre os diversos componentes da Atenção Básica, Especializada e Hospitalar foi justificada por alguns sujeitos da pesquisa devido à grande demanda dos trabalhadores em seus locais de atuação: "Então, hoje. o que eu vejo, é cada um fazendo o seu trabalho, devido ao fluxo grande de cada um na sua área. E, nessa integração, a gente está pecando" (S1).

Ao mesmo tempo, ainda que incipientes, emergem alguns movimentos no sentido de favorecer essa integração, como reuniões de rede e encontros intersetoriais, de modo a promover um cuidado integral e articulado:

O que a gente está percebendo: aos pouquinhos a rede funciona melhor. Por exemplo, essa semana, eu já participei de uma reunião de rede, onde estavam os serviços todos envolvidos, saúde, assistência, em relação a uma demanda, enfim, a uma paciente. Então, aos pouquinhos vai indo, mas a gente está sempre ali não é, provocando, buscando. (T1).

Considerando as especificidades que a vivência da deficiência impõe, as necessidades das PcD vão além da saúde, sendo as ações intersetoriais imprescindíveis ao atendimento integral dessas pessoas ${ }^{15}$. A rede deve se caracterizar pela relação de interdependência que se estabelece entre seus componentes, devido aos interesses e objetivos comuns ${ }^{\mathbf{1 6}}$. Assim, torna-se necessária a coordenação entre diferentes profissionais e serviços para uma abordagem integral desses sujeitos, de maneira que a fusão de ações promova a qualidade de vida e a inclusão das $\mathrm{PcD}$ na sociedade ${ }^{17}$, respeitando as diversidades territoriais $\mathrm{e}$ culturais. Compreendeu-se que, mais do que uma diretriz formal da rede, essa articulação intra e entre redes depende de iniciativas e movimentos individuais dos trabalhadores que atuam nos diversos serviços, compondo, dessa maneira, uma rede viva e em constante mutação:

E uma coisa que eu percebo que depende das pessoas que estão nos serviços. Às vezes o serviço não participa, não vai, porque as pessoas que estão lá não percebem a importância disso; e outros têm as pessoas que fazem as coisas acontecerem. (T1).

Dessa maneira, valorizam-se os encontros ocorridos no cotidiano das relações, destacando-se a importância dos movimentos micropolíticos para a implantação da RCPCD e qualificação do cuidado.

\section{(Im)possibilidades da rede}
A figura 3 destaca as principais potências e fragilidades da RCPCD mencionadas pelos sujeitos do estudo: 
Figura 3. Potências e limitações da RCPCD na 28ª Região de Saúde, RS

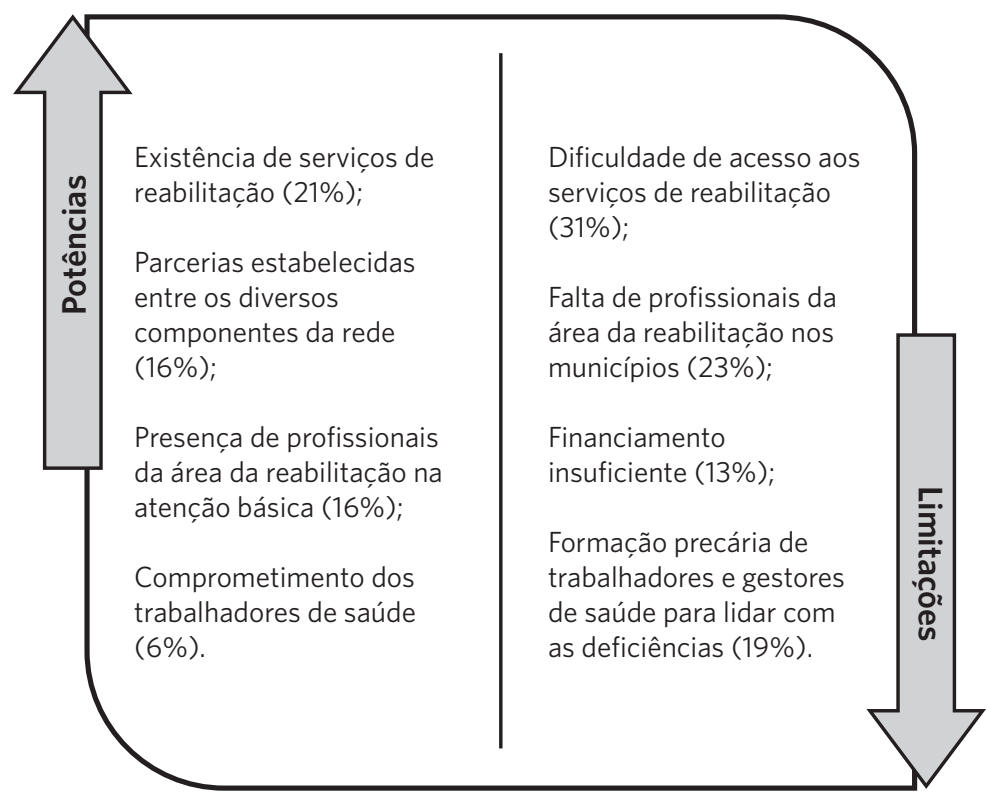

Dentre as potencialidades, a mais citada foi a existência de serviços de reabilitação de referência, ressaltando-se, assim, o lugar fundamental desses serviços na implementação da RCPCD na região estudada:

Bom, acho que a nossa região, ela é muito bem assessorada. Se não me falha a memória, ela tem todas as áreas pras PcD: a física, a intelectual, a auditiva não me lembro se tá aqui. (R2).

Esse reconhecimento dos serviços especializados de reabilitação pode ser decorrente do fato que, antes da atual política estimuladora do cuidado em rede, a maioria das ações em saúde voltadas às $\mathrm{PcD}$ estavam restritas a tais serviços vinculados à atenção especializada. Por outro lado, a dificuldade de acesso e as extensas filas de espera para esses serviços foi referida como o principal limitador na atenção à saúde das $\mathrm{PcD}$ na região:

Mesmo que pra gente pareça rápido, pra quem precisa continua sendo demorado. Acho que o desafio principal é diminuir as filas pros serviços de reabilitação. (B1).
Inúmeros fatores estão envolvidos na problemática do acesso à atenção especializada, existindo registros de longa espera para atendimento em algumas especialidades, além de outras questões que impossibilitam o seguimento do tratamento tais como a insuficiência de recursos financeiros para ampliação da capacidade de atendimento e a falta de transporte adaptado, sobretudo em se tratando de uma população com deficiência ou mobilidade reduzida ${ }^{13}$.

Para que a RCPCD cumpra seu papel, torna-se necessária uma clara definição e conhecimento público da missão assistencial de cada ponto de atenção, bem como dos fluxos entre os serviços ${ }^{18}$. Essa constatação coloca em discussão o lugar que a atenção básica ocupa no funcionamento das RAS, permitindo que se questione o seu papel como base do sistema, coordenadora do cuidado e ordenadora das redes ${ }^{19}$.

Nesse sentido, citou-se como potencialidade da rede em alguns municípios, a inserção de profissionais da área da reabilitação na atenção básica, como fisioterapeutas e fonoaudiólogos, seja na composição de equipes 
mínimas ou compondo os Nasf. A inclusão de tais profissionais nesse nível de atenção possibilita uma relação muito próxima com os usuários, especialmente aqueles com alguma deficiência, permitindo identificar riscos e possibilidades de intervenções da equipe junto aos indivíduos e suas famílias baseados em um cuidado à saúde que respeite a dimensão dialógica do encontro ${ }^{20}$. Assim, o atendimento desses usuários no nível de atenção básica, quando possível, pode representar um elemento facilitador do acesso ao cuidado pelas PcD, diminuindo a demanda reprimida nos setores especializados em função do modelo assistencial tradicional que não permite a integralidade na atenção às pessoas com deficiência.

Para isso, é preciso, além da inclusão desses profissionais, a efetivação de novos dispositivos e tecnologias para o cuidado em saúde. Uma potente tática para superar a dificuldade de acesso e ampliar as possibilidades de atenção a esses sujeitos pode ser a utilização da ferramenta do Apoio Matricial, a ser desenvolvido tanto pelos serviços de reabilitação como pelos Nasf. O Apoio Matricial traz uma proposta inovadora e potente para a transformação do modelo assistencial, alterando metodologias de trabalho por meio de mecanismos de personalização, diálogo, decisão compartilhada, responsabilização e compromisso entre equipes e apoiadores ${ }^{21}$.

Em contrapartida, a contratação desses profissionais ainda é uma realidade distante para muitos locais, sendo a ausência de profissionais da área de reabilitação nos municípios fator limitante para a atenção integral. Vale observar que os municípios estudados são, em sua maioria, de pequeno porte, e, segundo alguns gestores de saúde, a inserção de tais profissionais originaria mais despesas: "Então, tudo esbarra na burocracia, porque espaço físico, temos bastante, mas precisamos dos profissionais. Profissional tem custo, não é?" (S1).

Nesse sentido, um grande empecilho para a efetiva implementação da RCPCD, sobretudo para gestores e coordenadores de serviços, é a insuficiência de recursos financeiros para investimentos, execução de ações e, principalmente, para a ampliação das equipes de saúde:

O nosso maior problema hoje é financeiro, nós teríamos que contratar mais uma fonoaudióloga, teríamos que contratar mais uma fisioterapeuta, mais uma terapeuta ocupacional; existe demanda, mas a situação financeira não nos permite. (R1).

Além de insuficientes, frequentemente os recursos do SUS não estão alinhados com os objetivos das RAS, havendo predomínio das formas de pagamento por procedimentos que incentivam a prestação de serviços de maior densidade tecnológica em detrimento de serviços mais efetivos em custo e necessários do ponto de vista sanitário. Assim, torna-se urgente incentivar modos de financiamento que priorizem os cuidados preventivos e a atenção que gere valor aos usuários ${ }^{22}$.

O comprometimento dos trabalhadores da rede também foi uma das potencialidades mencionadas, salientando que o foco na melhoria do processo de trabalho influencia positivamente o acolhimento e o cuidado integral a essas pessoas: "Pelo menos o que a gente tem percebido na saúde pública é a busca por parte dos profissionais de saúde em se inteirar dessa questão da deficiência” (P1).

Entretanto, os limites dos trabalhadores e gestores para lidar com as deficiências também foram destacados: "Às vezes, a gente vai atender, mas não sabe lidar com a dificuldade do outro e acaba ficando sem saber o que fazer" (B1).

Dessa maneira, a qualificação dos profissionais para trabalhar com PcD foi citada como uma importante ação para a melhoria da atenção à saúde dessas pessoas. Nesse contexto, ressaltou-se a necessidade de maior sensibilidade e humanização nos serviços de saúde, favorecendo o comprometimente de todos os envolvidos no cuidado às PcD, sobretudo dos profissionais de saúde, a 
fim de que se produzam práticas de cuidado mais humanizadas: "O que eu acho que falta é um pouco mais de sensibilidade nos serviços, de não ser só mais um número e sim de levar em consideração que é uma pessoa" (T1).

Ressalta-se a importância de atores com conhecimento e habilidades específicas implicados com a implantação das RAS na região, como os gestores públicos, técnicos, trabalhadores de saúde e usuários ${ }^{23}$. As possibilidades de promoção de estratégias de educação permanente dos profissionais que atuam com $\mathrm{PcD}$ merecem destaque, sendo, inclusive, uma das diretrizes para o funcionamento da rede de cuidados à pessoa com deficiência ${ }^{7}$.

Tanto quanto as articulações macropolíticas, devem ser considerados também os movimentos micropolíticos empregados na articulação dessa rede, sendo considerada como importante potencialidade as parcerias estabelecidas entre os diversos serviços e atores:

Mesmo com essas dificuldades todas, a gente tem bons serviços, a gente tem essa rede, esses contatos; então, quando se precisa realmente, a gente consegue agilizar. Os serviços estão, como vou dizer, abertos a essas demandas. (T1).

Os movimentos micropolíticos acontecem no agir do trabalho vivo, nos encontros em espaços formais e informais, entre os próprios trabalhadores, gestores e usuários, os quais produzem novos arranjos em suas ações, conversações e encontros ${ }^{24}$. Esses movimentos, apesar de não formalizados, dão vida e fluidez à rede de atenção. Assim sendo, as interações e tessituras que se dão no cotidiano das relações, reafirmam o pressuposto da existência de uma rede viva e em constante mutação.

Ao ampliar a compreensão sobre a complexa realidade da saúde de uma região, é possível elaborar projetos mais condizentes e potentes para intervir no sistema de saúde regional23. A implementação da RCPCD deve garantir a integralidade do cuidado por meio do fortalecimento e integração das ações de saúde em todos os seus componentes, além de colocar a agenda das PcD de modo transversal no SUS, usando as redes a favor do acolhimento e cuidado que essa população necessita, como direito inquestionável de todo cidadão brasileiro ${ }^{8}$.

\section{Considerações finais}

Uma rede que garanta a integralidade do cuidado às Pessoas com Deficiência precisa dispor de ações e serviços articulados entre si que favoreçam as parcerias entre os diversos serviços e atores da rede, o financiamento adequado, além do comprometimento de trabalhadores e gestores de saúde para lidar com as deficiências. Para que as necessidades de saúde dessas pessoas sejam efetivamente atendidas, é importante fortalecer a rede conforme diretrizes definidas e, ao mesmo tempo, respeitar e dar visibilidade aos movimentos singulares existentes, na condição de uma rede viva de cuidados, salientando que esta rede deve ser tecida nos encontros cotidianos entre gestores, trabalhadores e usuários.

A análise dos dados revelou uma RCPCD em construção na região estudada, onde ocorre processo gradual de incorporação de suas diretrizes no cuidado às $\mathrm{PcD}$. E, mais do que isso, percebeu-se que esta rede se configura como uma rede viva de cuidados em permanente movimento e se delineando conforme as necessidades da população com deficiência do território, criando arranjos próprios por mio de constantes movimentos macro e micropolíticos que intencionam contemplar a integralidade da atenção à saúde.

Destaca-se que as questões relativas à assistência às pessoas com deficiências física, intelectual e auditiva apareceram reiteradamente entre os sujeitos do estudo. A questão da deficiência visual não foi citada pelos sujeitos. Compreende-se, diante dessa constatação, a necessidade de se colocar em debate a saúde das pessoas com deficiência visual, pois, apesar de ser a deficiência que 
apresenta maior prevalência, tanto em âmbito regional como nacional, as políticas públicas de saúde voltadas a tais indivíduos ainda são ignoradas por grande parcela da população. Há de se considerar, também, que a região não possui serviço de referência para deficiência visual, o que contribui para o seu desconhecimento por grande parte de trabalhadores, gestores, usuários e serviços de saúde.

Dessa maneira, este estudo pode contribuir no sentido de considerar e valorizar uma 'rede viva de cuidados' em permanente movimento, além de promover discussões e reflexões sobre a temática da saúde das pessoas com deficiência, possibilitando a todos os atores reconhecer, repensar e aprimorar suas práticas, contribuindo para transformações e inovações no campo da saúde da pessoa com deficiência.

\section{Colaboradores}

CD, ELG e SBFK contribuíram substancialmente para a concepção, planejamento, análise e interpretação dos dados, bem como para elaboração do rascunho e revisão crítica do conteúdo.

\section{Referências}

1. Campos M, Souza LAP, Mendes VLF. A rede de cuidados do Sistema Único de Saúde à saúde das pessoas com deficiência. Interface. 2015; 19(52):207210.

2. World Health Organization. The World Bank. World report on disability. Genebra: WHO; 2011. [acesso em 2016 out 2]. Disponível em: http://www. who.int/disabilities/world_report/2011/report.pdf.

3. Instituto Brasileiro de Geografia e Estatística. Censo Demográfico 2010: Banco de Dados agregados do IBGE. Rio de Janeiro: IBGE; [data desconhecida] [acesso em 2015 jun 4]. Disponível em: http:// www.ibge.gov.br/home/estatistica/populacao/censo2010/default.shtm.

4. Feuerwerker LCM, Cecílio LCO. O hospital e a formação em saúde: desafios atuais. Ciênc. Saúde Colet. 2007; 12(4):965-971.
5. Cecílio LCO, Merhy EE. A integralidade do cuidado como eixo da gestão hospitalar. In: Pinheiro R, Mattos R. Construção da integralidade: cotidiano, saberes e práticas em saúde. Rio de Janeiro: IMS/Abrasco, 2003.

6. Brasil. Portaria $\mathrm{n}^{\circ} 4.279$, de 30 de dezembro de 2010. Estabelece diretrizes para a organização da Rede de Atenção à Saúde no âmbito do Sistema Único de Saúde (SUS). Diário Oficial da União. 30 dez 2010.

7. Brasil. Portaria $\mathrm{n}^{\circ} 793$, de 24 de abril de 2012. Institui a Rede de Cuidados à Pessoa com Deficiência no âmbito do Sistema Único de Saúde. Diário Oficial da União. 25 abr 2012.

8. Mendes VLF. Da 'narrativa da dificuldade' ao diálogo com a diferença: a construção da Rede de Cuidados à Saúde da Pessoa com Deficiência. In: Brasil. Ministério da Saúde. Diálogo (bio)político: sobre al- 
guns desafios da construção da Rede de Cuidados à Saúde da Pessoa com Deficiência. Brasília, DF: Ministério da Saúde; 2014. p. 37-68.

9. Hoffmann K, Stein KV, Maier M, et al. Access points to the different levels of health care and demographic predictors in a country without a gatekeeping system. Results of a cross-sectional study from Austria. Eur J Public Health. 2013; 23(6):933-939.

10. Minayo MCS. O desafio do conhecimento: pesquisa qualitativa em saúde. São Paulo: Hucitec; 2014.

11. Merhy EE, Gomes MPC, Silva E, et al. Redes Vivas: multiplicidades girando as existências, sinais da rua. Implicações para a produção do cuidado e a produção do conhecimento em saúde. Saúde debate. $2014 ;$ 52:153-164.

12. Abrahão AL, Merhy EE, Cerqueira Gomes MP, et al. O pesquisador IN-MUNDO e o processo de produção de outras formas de investigação em saúde. In: Cerqueira Gomes MP, Merhy EE, organizadores. Pesquisadores IN-MUNDO: um estudo da produção do acesso e barreira em saúde mental. Porto Alegre: Rede Unida; 2014. p.155-170.

13. Othero MB, Dalmaso ASW. Pessoas com deficiência na atenção primária: discurso e prática de profissionais em um centro de saúde-escola. Interface. 2009; 13(28):177-188.

14. Brasil. Secretaria de Atenção à Saúde. Ministério da Saúde. Núcleo Técnico da Política Nacional de Humanização. HumanizaSUS: Documento base para gestores e trabalhadores do SUS. 4. ed. Brasília, DF: MS; 2008

15. Othero MB, Ayres JRCM. Necessidades de saúde da pessoa com deficiência: a perspectiva dos sujeitos por meio de histórias de vida. Interface. 2012; 16(40):219-233.

16. Santos L, Andrade LOM. Redes no SUS: marco legal. In: Silva SF. Redes de Atenção à Saúde: desafios da regionalização no SUS. Campinas: Saberes; 2013. p. 22-34.
17. Cilleros MVM, Gómez MCS. Análisis cualitativo de tópicos vinculados a la calidad de vida en personas con discapacidad. Ciênc. Saúde Colet. 2016; 21(8):2365-2374

18. Magalhães Junior HM. Redes de Atenção à Saúde: rumo à integralidade. Saúde debate. 2014; 52:15-37.

19. Brasil. Portaria $\mathrm{n}^{\circ} 2.488$, de 21 de outubro de 2011. Aprova a Política Nacional de Atenção Básica, estabelecendo a revisão de diretrizes e normas para a organização da Atenção Básica, para a Estratégia Saúde da Família (ESF) e o Programa de Agentes Comunitários de Saúde (PACS). Diário Oficial da União. 21 out 2011.

20. Vieira DKR, Favoreto CAO. Narrativas em saúde: refletindo sobre o cuidado à pessoa com deficiência $\mathrm{e}$ doença genética no Sistema Único de Saúde (SUS). Interface. 2016; 20(56):89-98.

21. Castro CP, Campos GWS. Apoio Matricial como articulador das relações interprofissionais entre serviços especializados e atenção primária à saúde. Physis. 2016; 26(2):455-481.

22. Mendes EV. Comentários sobre as Redes de Atenção à Saúde no SUS. Saúde debate 2014; 52:38-49.

23. Silva SF. Contribuição para a análise da implantação de Redes de Atenção à Saúde no SUS. Saúde debate. 2014; 52:165-176.

24. Merhy EE. Educação Permanente em Movimento - uma política de reconhecimento e cooperação, ativando os encontros do cotidiano no mundo do trabalho em saúde, questões para os gestores, trabalhadores e quem mais quiser se ver nisso. Saúde Redes. $2015 ; 1(1): 7-14$.

Recebido em 03/01/2018

Aprovado em 08/05/2018

Conflito de interesses: inexistente

Suporte financeiro: não houve 